\title{
SYMMETRY BREAKING FOR GROUND-STATE SOLUTIONS OF HÉNON SYSTEMS IN A BALL
}

\author{
HAIYANG HE \\ Department of Mathematics, Hunan Normal University, Changsha Hunan 410081, \\ The People's Republic of China \\ e-mail: hehy917@yahoo.com.cn
}

(Received 3 June 2009; revised 9 January 2010; accepted 28 June 2010; first published online 8 December 2010)

Abstract. We consider in this paper the problem

$$
\begin{cases}-\Delta u=|x|^{\alpha} v^{p}, & x \in \Omega, \\ -\Delta v=|x|^{\beta} u^{q_{\varepsilon}}, & x \in \Omega, \\ u>0, \quad v>0, & x \in \Omega, \\ u=v=0, & x \in \partial \Omega,\end{cases}
$$

where $\Omega$ is the unit ball in $\mathbb{R}^{N}$ centred at the origin, $0 \leq \alpha<p N, \quad \beta>0, \quad N \geq 3$. Suppose $q_{\varepsilon} \rightarrow q$ as $\varepsilon \rightarrow 0^{+}$and $q_{\varepsilon}, q$ satisfy, respectively,

$$
\frac{N}{p+1}+\frac{N}{q_{\varepsilon}+1}>N-2, \quad \frac{N}{p+1}+\frac{N}{q+1}=N-2 ;
$$

we investigate the asymptotic estimates of the ground-state solutions $\left(u_{\varepsilon}, v_{\varepsilon}\right)$ of $(1)$ as $\beta \rightarrow+\infty$ with $p, q_{\varepsilon}$ fixed. We also show the symmetry-breaking phenomenon with $\alpha, \beta$ fixed and $q_{\varepsilon} \rightarrow q$ as $\varepsilon \rightarrow 0^{+}$. In addition, the ground-state solution is non-radial provided that $\varepsilon>0$ is small or $\beta$ is large enough.

2010 Mathematics Subject Classification. 35J50, 35J60.

1. Introduction. In this paper, we investigate the limiting behaviour of the groundstate solutions of the problem

$$
\begin{cases}-\Delta u=|x|^{\alpha} v^{p}, & x \in \Omega, \\ -\Delta v=|x|^{\beta} u^{q_{\varepsilon}}, & x \in \Omega, \\ u=v=0, & x \in \partial \Omega,\end{cases}
$$

where $\Omega$ is the unit ball in $\mathbb{R}^{N}$ centred at the origin, $0 \leq \alpha<p N, \beta>0, N \geq 3$. We assume in this paper that $q_{\varepsilon} \rightarrow q$ as $\varepsilon \rightarrow 0^{+}$and $q_{\varepsilon}, q$ satisfy, respectively,

$$
\frac{N}{p+1}+\frac{N}{q_{\varepsilon}+1}>N-2, \quad \frac{N}{p+1}+\frac{N}{q+1}=N-2 .
$$

Problem (2) has two features. First, it is a Hénon-type system. The Hénon equation with Dirichlet boundary conditions

$$
\begin{cases}-\Delta u=|x|^{\alpha} u^{p}, & x \in \Omega, \\ u=0, & x \in \partial \Omega\end{cases}
$$


was found in [10], which stems from rotating stellar structures. A standard compactness argument show that the infimum

$$
\inf _{u \in H_{0}^{1}(\Omega) \backslash\{0\}} \frac{\int_{\Omega}|\nabla u|^{2} d x}{\left(\int_{\Omega}|x|^{\alpha}|u|^{p+1} d x\right)^{\frac{2}{p+1}}}
$$

is achieved for any $1<p<2^{*}-1, \alpha>0$. In 1982, $\mathrm{Ni}[\mathbf{1 4}]$ proved that the infimum

$$
\inf _{u \in H_{0, \text { rad }}^{1}(\Omega) \backslash\{0\}} \frac{\int_{\Omega}|\nabla u|^{2} d x}{\left(\int_{\Omega}|x|^{\alpha}|u|^{p+1} d x\right)^{\frac{2}{p+1}}}
$$

is achieved for any $p \in\left(1, \frac{N+2+2 \alpha}{N-2}\right)$ by a function in $H_{0, \text { rad }}^{1}(\Omega)$, the space of radial $H_{0}^{1}(\Omega)$ functions. Thus, radial solutions of (3) exist also for (Sobolev) supercritical exponents $p$. A natural question is whether any minimizer of (4) must be radially symmetric in the range $1<p<\frac{N+2}{N-2}$ and $\alpha>0$. Since the weight $|\cdot|^{\alpha}$ is an increasing function, neither rearrangement arguments nor the moving plane techniques of [7] can be applied.

For $\alpha>0$, Smets et al. proved in [15] some symmetry-breaking results for (3). They proved that the minimizers of (4) (the so-called ground-state solutions, or least energy solutions) cannot be radial for $\alpha$ large enough. As a consequence, (3) has at least two solutions when $\alpha$ is sufficiently large (see also [16]).

Quite recently, Cao and Peng [3] proved that for $p+1$ sufficiently close to $2^{*}$, the ground-state solutions of (3) possess a unique maximum point whose distance from $\partial \Omega$ tends to zero as $p \rightarrow \frac{N+2}{N-2}$.

For more results about symmetry breaking phenomena for solutions of problem (3) either $\alpha$ is large enough or $p \rightarrow \frac{N+2}{N-2}$, see for instance $[2,1]$ and references therein.

Second, the system in (2) is a Hamiltonian-type system, which is strongly indefinite. The existence of solutions of the Hamiltonian elliptic system

$$
\begin{cases}-\Delta u=v^{p}, & x \in \Omega, \\ -\Delta v=u^{q}, & x \in \Omega, \\ u=v=0, & x \in \partial \Omega\end{cases}
$$

was first considered in [5] and [11] with $\frac{1}{p+1}+\frac{1}{q+1}>\frac{N-2}{N}$; the curve of $(p, q) \in \mathbb{R}^{2}$ satisfying $\frac{1}{p+1}+\frac{1}{q+1}=\frac{N-2}{N}$ is called critical hyperbola. Afterwards, various results were obtained in the literature. Extensions of problem (6) can be found in [6] and [13]. In [13], existence problems for Hardy-type systems and Hénon-type systems were established. Particularly, for Hénon-type systems

$$
\begin{cases}-\Delta u=|x|^{\alpha} v^{p}, & x \in \Omega, \\ -\Delta v=|x|^{\beta} u^{q}, & x \in \Omega, \\ u=v=0, & x \in \partial \Omega,\end{cases}
$$

the critical hyperbola is $\frac{1}{p+1}\left(1+\frac{\alpha}{N}\right)+\frac{1}{q+1}\left(1+\frac{\beta}{N}\right)=\frac{N-2}{N}$.

In recent years, a study of the limiting behaviour of ground-state solutions of elliptic problems has attracted considerable attention. For the system (6), the limiting behaviour of solutions of (6) as $\frac{1}{p+1}+\frac{1}{q+1} \rightarrow \frac{N-2}{N}$ was discussed in [8]. For the system (7), Yang and He [9] proved that for $\frac{1}{p+1}+\frac{1}{q+1} \rightarrow \frac{N-2}{N}$, the ground-state solutions of (7) possess a unique maximum point whose distance from $\partial \Omega$ tends to zero as 
$\frac{1}{p+1}+\frac{1}{q+1} \rightarrow \frac{N-2}{N}$. Such problems are closely related to solutions of the following problem:

$$
\begin{cases}-\Delta U=V^{p}, & y \in \mathbb{R}^{N}, \\ -\Delta V=U^{q}, & y \in \mathbb{R}^{N}, \\ U(y)>0, \quad V(y)>0, & y \in \mathbb{R}^{N}, \\ U(0)=1, \quad U \rightarrow 0, \quad V \rightarrow 0 \text { as }|y| \rightarrow \infty, & \end{cases}
$$

where $\frac{1}{p+1}+\frac{1}{q+1}=\frac{N-2}{N}$. It was proved in [12] that $U \in D^{2, \frac{p+1}{p}}\left(\mathbb{R}^{N}\right), \quad V \in D^{2, \frac{q+1}{q}}\left(\mathbb{R}^{N}\right)$, where $D^{2, r}\left(\mathbb{R}^{N}\right)$ is the completion of $C_{0}^{\infty}\left(\mathbb{R}^{N}\right)$ with respect to the norm $\|\Delta \cdot\|_{r}$. Actually, $U$ and $V$ are radially symmetric for $p \geq 1$ as showed in [4]. Moreover, $U$ and $V$ are unique and decreasing in $r$. In the discussion of one equation problem, one uses the instanton for the best Sobolev constant. However, no explicit form of $(U, V)$ was found for $p>\frac{2}{N-2}$ up to now. Instead, the asymptotic behaviour of $(U, V)$ as $r \rightarrow \infty$ is sufficient for this purpose. It was found in [12] that

$$
\lim _{r \rightarrow \infty} r^{N-2} V(r)=a, \quad \begin{cases}\lim _{r \rightarrow \infty} r^{N-2} U(r)=b & \text { if } p>\frac{N}{N-2}, \\ \lim _{r \rightarrow \infty} \frac{r^{N-2}}{\log r} U(r)=b & \text { if } p=\frac{N}{N-2}, \\ \lim _{r \rightarrow \infty} r^{p(N-2)-2} U(r)=b & \text { if } \frac{2}{N-2}<p<\frac{N}{N-2},\end{cases}
$$

and

$$
\lim _{r \rightarrow \infty} \frac{r V^{\prime}(r)}{V(r)}=2-N, \quad \begin{cases}\lim _{r \rightarrow \infty} \frac{r U^{\prime}(r)}{U(r)}=2-N & \text { if } p \geq \frac{N}{N-2}, \\ \lim _{r \rightarrow \infty} \frac{r U^{\prime}(r)}{U(r)}=2-p(N-2) & \text { if } p \leq \frac{N}{N-2} .\end{cases}
$$

In this paper, we are interested in the symmetry of ground-states solutions of (2). Now, we denote

$$
E_{\alpha}(\Omega)=\left\{u \in W^{2, \frac{p+1}{p}} \cap W_{0}^{1, \frac{p+1}{p}}(\Omega): \int_{\Omega}|x|^{-\frac{\alpha}{p}}|\Delta u|^{\frac{p+1}{p}} d x<\infty\right\}
$$

and

$$
E_{\alpha}^{\mathrm{rad}}(\Omega)=\left\{u \in E_{\alpha}(\Omega): u(x)=u(|x|)\right\}
$$

Our main results are as follows.

TheOREM 1.1. Suppose $N \geq 3,0 \leq \alpha<p N, \beta>0, p>\frac{2}{N-2}, q_{\varepsilon}>\frac{N+p}{N p-2 p-1}$; then there exists $\beta^{*}>0$ such that the ground-state solutions $u_{\alpha, \beta, \varepsilon}$ are non-radial provided $\beta>\beta^{*}$.

TheOREM 1.2. Suppose $N \geq 3,0 \leq \alpha<p N, \beta>0, p>\frac{2}{N-2}, p q_{\varepsilon}>1$; then there exists $\varepsilon^{*}>0$ such that the ground-state solutions $u_{\alpha, \beta, \varepsilon}$ are non-radial provided $\varepsilon<\varepsilon^{*}$ or $q-q_{\varepsilon}<\varepsilon^{*}$.

This paper is organized as follows. In section 2, we give some preliminaries which turn out to be essential. In section 3 , we present some estimates for radial groundstate solutions of (2) with $\alpha, p, q_{\varepsilon}$ fixed and $\beta \rightarrow \infty$. This will lead us to get the first 
symmetry-breaking result, stating that for $\beta$ sufficiently large, the ground-state solution of problem (2) is non-radial. In section 4, another symmetry-breaking result is proved, with $\alpha, \beta, p$ fixed and $q_{\varepsilon} \rightarrow q$ as $\varepsilon \rightarrow 0$.

2. Preliminaries. Before proving our main results, we want to introduce some simple calculus lemma which turns out to be essential:

LEMMA 2.1. Let $u$ be a radially symmetric function of $\Omega$ (unit ball in $\mathbb{R}^{N}$ ) with $u(1)=0, u^{\prime}(0)$ exists. Then

$$
\begin{aligned}
\text { (i) }|u(x)| & \leq \frac{1}{w_{N-1}^{\frac{p}{p+1}}(p(N-1)-1)^{\frac{1}{p+1}}} \frac{\left(\int_{\Omega}|\nabla u|^{\frac{p+1}{p}} d y\right)^{\frac{p}{p+1}}}{\left(|x|^{p(N-1)-1}\right)^{\frac{1}{p+1}}} \\
\text { (ii) }\left|\frac{\partial u}{\partial r}\right| & \leq r^{1-N}\left(\frac{r^{N+\alpha}}{N+\alpha}\right)^{\frac{1}{p+1}}\left(\frac{1}{w_{N-1}} \int_{B_{r}(0)}|x|^{-\frac{\alpha}{p}}|\Delta u|^{\frac{p+1}{p}} d x\right)^{\frac{p}{p+1}},
\end{aligned}
$$

where $w_{N-1}$ is the surface area of the unit ball in $\mathbb{R}^{N}$.

Proof. (i) For

$$
\begin{aligned}
u(1)-u(x) & =\int_{|x|}^{1}\left|u^{\prime}(t)\right| d t \\
|u(x)| & \leq \int_{|x|}^{1}\left|u^{\prime}(t)\right| d t \\
& \leq\left(\int_{|x|}^{1}\left|u^{\prime}(t)\right|^{\frac{p+1}{p}} t^{N-1} d t\right)^{\frac{p}{p+1}}\left(\int_{|x|}^{1} t^{-(N-1) p} d t\right)^{\frac{1}{p+1}} .
\end{aligned}
$$

Since

$$
\int_{|x|}^{1} t^{-(N-1) p} d t=\frac{1}{p(N-1)-1}\left(\frac{1}{|x|^{p(N-1)-1}}-1\right) \leq \frac{1}{p(N-1)-1} \frac{1}{|x|^{p(N-1)-1}}
$$

and

$$
\begin{aligned}
\int_{|x|}^{1}\left|u^{\prime}(t)\right|^{\frac{p+1}{p}} t^{N-1} d t & =\frac{1}{w_{N-1}} \int\left(\int_{|x|}^{1}\left|u^{\prime}(t)\right|^{\frac{p+1}{p}} t^{N-1} d t\right) w(\theta) d \theta \\
& =\frac{1}{w_{N-1}} \int_{|x| \leq|y| \leq 1}|\nabla u|^{\frac{p+1}{p}} d y \\
& \leq \frac{1}{w_{N-1}} \int_{\Omega}|\nabla u|^{\frac{p+1}{p}} d y
\end{aligned}
$$

we find that

$$
|u(x)| \leq \frac{1}{w_{N-1}^{\frac{p}{p+1}}(p(N-1)-1)^{\frac{1}{p+1}}} \frac{\left(\int_{\Omega}|\nabla u|^{\frac{p+1}{p}} d y\right)^{\frac{p}{p+1}}}{\left(|x|^{p(N-1)-1}\right)^{\frac{1}{p+1}}} .
$$


(ii) For

$$
\begin{aligned}
\left|r^{N-1} \frac{\partial u}{\partial r}\right| & \leq \int_{0}^{r} t^{N-1}|\Delta u| d t \\
& \leq\left(\int_{0}^{r} t^{\frac{(N-1+\alpha)(p+1)}{p+1}} d t\right)^{\frac{1}{p+1}}\left(\int_{0}^{r}\left(t^{\frac{p(N-1)-\alpha}{p+1}}|\Delta u|\right)^{\frac{p+1}{p}} d t\right)^{\frac{p}{p+1}} \\
& =\left(\frac{r^{N+\alpha}}{N+\alpha}\right)^{\frac{1}{p+1}}\left(\frac{1}{w_{N-1}} \int_{B_{r}(0)}|x|^{-\frac{\alpha}{p}}|\Delta u|^{\frac{p+1}{p}} d x\right)^{\frac{p}{p+1}}
\end{aligned}
$$

we have

$$
\left|\frac{\partial u}{\partial r}\right| \leq r^{1-N}\left(\frac{r^{N+\alpha}}{N+\alpha}\right)^{\frac{1}{p+1}}\left(\frac{1}{w_{N-1}} \int_{\Omega}|x|^{-\frac{\alpha}{p}}|\Delta u|^{\frac{p+1}{p}} d x\right)^{\frac{p}{p+1}}
$$

As a result of Lemma 2.1, we obtain the following corollary.

COROLlary 2.1. Under hypothesis of Lemma 2.1, and if $\frac{N+\beta}{q+1}+\frac{N+\alpha}{p+1}>N-2$, then

$$
\int_{\Omega}|x|^{\beta}|u(x)|^{q+1} d x \leq C\left(\int_{\Omega}|x|^{-\frac{\alpha}{p}}|\Delta u|^{\frac{p+1}{p}} d x\right)^{\frac{p(q+1)}{p+1}}
$$

where

$$
C=\left(\frac{1}{w_{N-1}}\right)^{\frac{p(q+1)}{p+1}} \frac{1}{N+\beta-\left(N-2-\frac{N+\alpha}{p+1}\right)(q+1)}\left(\frac{1}{N-2-\frac{N+\alpha}{p+1}}\right)^{q+1}\left(\frac{1}{N+\alpha}\right)^{\frac{q+1}{p+1}}
$$

Proof. From the above Lemma 2.1, we have

$$
|u(|x|)| \leq \int_{|x|}^{1}\left|u^{\prime}(r)\right| d r \leq \int_{|x|}^{1}\left(\frac{r^{N+\alpha}}{N+\alpha}\right)^{\frac{1}{p+1}} r^{1-N} d r\left(\frac{1}{w_{N-1}} \int_{\Omega}|x|^{-\frac{\alpha}{p}}|\Delta u|^{\frac{p+1}{p}} d x\right)^{\frac{p}{p+1}} .
$$

Since

$$
\begin{aligned}
\int_{|x|}^{1}\left(\frac{r^{N+\alpha}}{N+\alpha}\right)^{\frac{1}{p+1}} r^{1-N} d r & =\left(\frac{1}{N+\alpha}\right)^{\frac{1}{p+1}} \int_{|x|}^{1} r^{\frac{N+\alpha}{p+1}} r^{1-N} d r \\
& \leq \frac{1}{N-2-\frac{N+\alpha}{p+1}}\left(\frac{1}{N+\alpha}\right)^{\frac{1}{p+1}} \frac{1}{|x|^{N-2-\frac{N+\alpha}{p+1}}}
\end{aligned}
$$

we have

$$
\begin{aligned}
& r^{\beta} r^{N-1}|u(r)|^{q+1} \\
& \leq r^{\beta} r^{N-1}\left(\frac{1}{N-2-\frac{N+\alpha}{p+1}}\left(\frac{1}{N+\alpha}\right)^{\frac{1}{p+1}} \frac{1}{r^{N-2-\frac{N+\alpha}{p+1}}}\right)^{q+1}\left(\frac{1}{w_{N-1}} \int_{\Omega}|x|^{-\frac{\alpha}{p}}|\Delta u|^{\frac{p+1}{p}} d x\right)^{\frac{p(q+1)}{p+1}}
\end{aligned}
$$


which implies

$$
\begin{aligned}
& \int_{0}^{1} r^{\beta} r^{N-1}|u(r)|^{q+1} d r \leq\left(\frac{1}{N-2-\frac{N+\alpha}{p+1}}\left(\frac{1}{N+\alpha}\right)^{\frac{1}{p+1}}\right)^{q+1} \\
& \quad \times \int_{0}^{1}\left(\frac{1}{r^{N-2-\frac{N+\alpha}{p+1}}}\right)^{q+1} r^{\beta} r^{N-1} d r\left(\frac{1}{w_{N-1}} \int_{\Omega}|x|^{-\frac{\alpha}{p}}|\Delta u|^{\frac{p+1}{p}} d x\right)^{\frac{p(q+1)}{p+1}}
\end{aligned}
$$

Thus, the conclusion holds.

3. Asymptotic estimates. Consider the minimization problem

$$
S_{\alpha, \beta, \varepsilon}^{\mathrm{rad}}=\inf _{u \in E_{\alpha}^{\mathrm{rad}}(\Omega) \backslash\{0\}} R_{\alpha, \beta, \varepsilon}(u),
$$

where

$$
R_{\alpha, \beta, \varepsilon}(u)=\frac{\int_{\Omega}|x|^{-\frac{\alpha}{p}}|\Delta u|^{\frac{p+1}{p}} d x}{\left(\int_{\Omega}|x|^{\beta}|u|^{q_{\varepsilon}+1} d x\right)^{\frac{p+1}{p\left(q_{\varepsilon}+1\right)}}}, \quad u \in E_{\alpha}(\Omega) \backslash\{0\},
$$

is the Rayleigh quotient associated with (2). Similar to [14], we can also prove that

$$
S_{\alpha, \beta, \varepsilon}^{\mathrm{rad}}(\Omega)=\inf _{u \in E_{\alpha}^{\mathrm{rad}}(\Omega) \backslash\{0\}} \frac{\int_{\Omega}|x|^{-\frac{\alpha}{p}}|\Delta u|^{\frac{p+1}{p}} d x}{\left(\int_{\Omega}|x|^{\beta}|u|^{q_{\varepsilon}+1} d x\right)^{\frac{p+1}{p\left(q_{\varepsilon}+1\right)}}}
$$

is attained by some positive function $u_{\alpha, \beta, \varepsilon}^{\mathrm{rad}}$. After scaling, $u_{\alpha, \beta, \varepsilon}^{\mathrm{rad}}$ is also a solution of (2).

Now, we provide an estimate of the energy $S_{\alpha, \beta, \varepsilon}^{\mathrm{rad}}$ as $\beta \rightarrow \infty$.

LEMMA 3.1. If $N \geq 3$, there exists $C>0$ depending on $N$, p such that

$$
S_{\alpha, \beta, \varepsilon}^{\mathrm{rad}} \geq C \beta^{\frac{p+2+q_{\varepsilon}}{p\left(q_{\varepsilon}+1\right)}} \text { as } \beta \rightarrow \infty
$$

Proof. Let $u \in E_{\alpha}^{\mathrm{rad}}(\Omega)$ and define the rescaled function $v(|x|)=u\left(|x|^{s}\right)$, where $s=\frac{N}{\beta+N}$. Then

$$
\int_{\Omega}|x|^{\beta}|u|^{q_{\varepsilon}+1} d x=w_{N-1} \int_{0}^{1} r^{\beta+N-1}|u(r)|^{q_{\varepsilon}+1} d r=s \int_{\Omega}|x|^{s(\beta+N)-N}|v(x)|^{q_{\varepsilon}+1} d x,
$$

and from Lemma 2.1, we have

$$
\int_{\Omega}|x|^{-\frac{\alpha}{p}}|\Delta u|^{\frac{p+1}{p}} d x \geq w_{N-1}^{\frac{p+1}{p}}(N+\alpha)^{\frac{1}{p}} r^{\frac{(p+1)(N-1)-(N+\alpha)}{p}}\left|\frac{\partial u}{\partial r}\right|^{\frac{p+1}{p}}
$$


which implies that

$$
\begin{aligned}
\int_{\Omega}|x|^{-\frac{\alpha}{p}}|\Delta u|^{\frac{p+1}{p}} d x & \geq w_{N-1}^{\frac{p+1}{p}}(N+\alpha)^{\frac{1}{p}} \int_{0}^{1} r^{\frac{(p+1)(N-1)-(N+\alpha)}{p}}\left|\frac{\partial u}{\partial r}\right|^{\frac{p+1}{p}} d r \\
& =w_{N-1}^{\frac{p+1}{p}}(N+\alpha)^{\frac{1}{p}} \int_{0}^{1} r^{\frac{(p+1)(N-1)-(N+\alpha)}{p}} s^{-\frac{p+1}{p}} r^{\frac{1-s}{s} \frac{p+1}{p}}\left|\frac{\partial v}{\partial t}\right|^{\frac{p+1}{p}} d r \\
& =w_{N-1}^{\frac{1}{p}}(N+\alpha)^{\frac{1}{p}} s^{-\frac{1}{p}} \int_{\Omega}|x|^{\frac{p(N-1)(s-1)-s(1+\alpha)+(1-s)}{p}}|\nabla v|^{\frac{p+1}{p}} d x .
\end{aligned}
$$

Thus, we obtain

$$
\frac{\int_{\Omega}|x|^{-\frac{\alpha}{p}}|\Delta u|^{\frac{p+1}{p}} d x}{\left(\int_{\Omega}|x|^{\beta}|u|^{q_{\varepsilon}+1} d x\right)^{\frac{p+1}{p\left(q_{\varepsilon}+1\right)}}} \geq \frac{w_{N-1}^{\frac{1}{p}}(N+\alpha)^{\frac{1}{p}} s^{-\frac{1}{p}} \int_{\Omega}|x|^{\frac{p(N-1)(s-1)-s(1+\alpha)+(1-s)}{p}}|\nabla v|^{\frac{p+1}{p}} d x}{\left(s \int_{\Omega}|v(x)|^{q_{\varepsilon}+1} d x\right)^{\frac{p+1}{p\left(q_{\varepsilon}+1\right)}}} .
$$

It follows that

$$
S_{\alpha, \beta, \varepsilon}^{\mathrm{rad}} \geq w_{N-1}^{\frac{1}{p}}(N+\alpha)^{\frac{1}{p}} S^{-\frac{1}{p}} s^{-\frac{p+1}{p\left(q_{\varepsilon}+1\right)}} \inf _{v \in W_{0, \text { rad }}^{1, \frac{p+1}{p}}}(\Omega) \quad \frac{\int_{\Omega}|x|^{\frac{p(N-1)(s-1)-s(1+\alpha)+(1-s)}{p}}|\nabla v|^{\frac{p+1}{p}} d x}{\left(\int_{\Omega}|v(x)|^{q_{\varepsilon}+1} d x\right)^{\frac{p+1}{p\left(q_{\varepsilon}+1\right)}}} .
$$

Now, we claim that for every $0 \leq s \leq 1$, we have $p(N-1)(s-1)-s(1+\alpha)+$ $(1-s)<0$. Indeed, for

$$
\begin{aligned}
& p(N-1)(s-1)-s(1+\alpha)+(1-s) \\
& \quad=(p(N-1)-2-\alpha) s-(p(N-1)-1),
\end{aligned}
$$

if $p(N-1)-2 \leq \alpha<p N$, we have $p(N-1)(s-1)-s(1+\alpha)+(1-s)<0$; if $\alpha<$ $p(N-1)-2<p(N-1)-1$, then for every $0 \leq s \leq 1$, we also have $p(N-1)(s-1)-$ $s(1+\alpha)+(1-s)<0$. Therefore,

$$
\int_{\Omega}|\nabla v|^{\frac{p+1}{p}} d x \leq \int_{\Omega}|x|^{\frac{p(N-1)(s-1)-s(1+\alpha)+(1-s)}{p}}|\nabla v|^{\frac{p+1}{p}} d x,
$$

which implies that

$$
c_{s}=\inf _{v \in W_{0, \text { rad }}^{1} \frac{p+1}{p}} \frac{\int_{\Omega}|x|^{\frac{p(N-1)(s-1)-s(1+\alpha)+(1-s)}{p}}|\nabla v|^{\frac{p+1}{p}} d x}{\left(\int_{\Omega}|v(x)|^{q_{\varepsilon}+1} d x\right)^{\frac{p+1}{p\left(q_{\varepsilon}+1\right)}}}
$$

is achieved by standard arguments. Since $|x| \leq 1$, if $p(N-1)-2 \leq \alpha<p N$, $p(N-1)(s-1)-s(1+\alpha)+(1-s) \leq-(p(N-1)-1)$, which implies that $c_{s} \geq$ $c_{0}$; if $\alpha<p(N-1)-2, p(N-1)(s-1)-s(1+\alpha)+(1-s)=(p(N-1)-2-\alpha) s-$ $(p(N-1)-1)$, then for every $0 \leq s \leq 1,|x|^{\frac{p(N-1)(s-1)-s(1+\alpha)+(1-s)}{p}}$ is non-increasing, which implies that $c_{s}$ is non-increasing on $[0,1]$; then $c_{s} \geq c_{1}$. Thus,

$$
S_{\alpha, \beta, \varepsilon}^{\mathrm{rad}} \geq C(N+\alpha)^{\frac{1}{p}} S^{-\frac{p+2+q_{\varepsilon}}{p\left(q_{\varepsilon}+1\right)}}, \quad \beta \rightarrow \infty .
$$


By the assumptions on $p, q_{\varepsilon}$, the inclusion $W^{2, \frac{p+1}{p}}(\Omega) \hookrightarrow L^{q_{\varepsilon}+1}(\Omega)$ is compact. It implies that

$$
S_{\alpha, \beta, \varepsilon}(\Omega)=\inf _{u \in E_{\alpha}(\Omega) \backslash\{0\}} \frac{\int_{\Omega}|x|^{-\frac{\alpha}{p}}|\Delta u|^{\frac{p+1}{p}} d x}{\left(\int_{\Omega}|x|^{\beta}|u|^{q_{\varepsilon}+1} d x\right)^{\frac{p+1}{p\left(q_{\varepsilon}+1\right)}}}
$$

is attained by some positive function $u_{\alpha, \beta, \varepsilon}$. After scaling, $u_{\alpha, \beta, \varepsilon}$ is a solution of (2).

LeMma 3.2. Assume $N \geq 3$, for any $p, q_{\varepsilon}$ satisfy $\frac{N}{p+1}+\frac{N}{q_{\varepsilon}+1}>N-2$ with $p>$ $\frac{2}{N-2}, q_{\varepsilon}>\frac{N+p}{N p-2 p-1}$; there exists $\beta^{*} \geq 0$ such that $S_{\alpha, \beta, \varepsilon}<S_{\alpha, \beta, \varepsilon}^{\mathrm{rad}}$ provided $\beta>\beta^{*}$.

Proof. For any fixed $u \in C_{0}^{\infty}(\Omega)$, define $u_{\beta}(x)=u\left(\beta\left(x-x_{\beta}\right)\right)$, where $x_{\beta}=(1-$ $\left.\frac{1}{\beta}, 0, \ldots, 0\right)$. For $\left|\beta\left(x-x_{\beta}\right)\right| \leq 1$, that is, $\left|x-x_{\beta}\right| \leq \frac{1}{\beta}$, then $|x| \geq\left|x_{\beta}\right|-\frac{1}{\beta}=1-\frac{2}{\beta}$. One has

$$
\int_{\Omega}|x|^{-\frac{\alpha}{p}}\left|\Delta u_{\beta}\right|^{\frac{p+1}{p}} d x \leq\left(1-\frac{2}{\beta}\right)^{-\frac{\alpha}{p}} \beta^{\frac{2(p+1)}{p}-N} \int_{\Omega}|\Delta u|^{\frac{p+1}{p}} d x,
$$

and

$$
\int_{\Omega}|x|^{\beta}\left|u_{\beta}\right|^{q_{\varepsilon}+1} d x \geq\left(1-\frac{2}{\beta}\right)^{\beta} \beta^{-N} \int_{\Omega}|u|^{q_{\varepsilon}+1} d x .
$$

Hence by definition, one obtains

$$
\begin{aligned}
S_{\alpha, \beta, \varepsilon} & \leq \frac{\int_{\Omega}|x|^{-\frac{\alpha}{p}}\left|\Delta u_{\beta}\right|^{\frac{p+1}{p}} d x}{\left(\int_{\Omega}|x|^{\beta}\left|u_{\beta}\right|^{q_{\varepsilon}+1} d x\right)^{\frac{p+1}{\left(q q_{\varepsilon}+1\right)}}} \\
& \leq \frac{\left(1-\frac{2}{\beta}\right)^{-\frac{\alpha}{p}} \beta^{\frac{2(p+1)}{p}-N} \int_{\Omega}|\Delta u|^{\frac{p+1}{p}} d x}{\left(\left(1-\frac{2}{\beta}\right)^{\beta} \beta^{-N} \int_{\Omega}|u|^{q_{\varepsilon}+1} d x\right)^{\frac{p+1}{p\left(q_{\varepsilon}+1\right)}}} \\
& \leq C_{1} \beta^{\frac{2(p+1)}{p}-N+\frac{N(p+1)}{p\left(q_{\varepsilon}+1\right)}} \frac{\int_{\Omega}|\Delta u|^{\frac{p+1}{p}} d x}{\left(\int_{\Omega}|u|^{q_{\varepsilon}+1} d x\right)^{\frac{p+1}{p\left(q_{\varepsilon}+1\right)}}} .
\end{aligned}
$$

Since $u$ is fixed and $\frac{\int_{\Omega}|\Delta u|^{\frac{p+1}{p}} d x}{\left(\int_{\Omega}|u|^{q_{\varepsilon}+1} d x\right)^{\frac{p+1}{\left(q_{\varepsilon}+1\right)}}}$ is independent of $\beta$, we have

$$
S_{\alpha, \beta, \varepsilon} \leq C \beta^{\frac{2(p+1)}{p}-N+\frac{N(p+1)}{p\left(q_{\varepsilon}+1\right)}} .
$$

From Lemma 3.1 $S_{\alpha, \beta, \varepsilon}^{\mathrm{rad}} \geq C \beta^{\frac{p+2+q_{\varepsilon}}{p\left(q_{\varepsilon}+1\right)}}$ as $\beta \rightarrow \infty$, and $\frac{p+2+q_{\varepsilon}}{p\left(q_{\varepsilon}+1\right)}>\frac{2(p+1)}{p}-N+\frac{N(p+1)}{p\left(q_{\varepsilon}+1\right)}$, that is, $q_{\varepsilon}>\frac{N+p}{N p-2 p-1}$. Hence $S_{\alpha, \beta, \varepsilon}<S_{\alpha, \beta, \varepsilon}^{\mathrm{rad}}$ as $\beta \rightarrow \infty$.

4. Analysis for $\varepsilon$ close to 0 . In this section, we analyse the case where $\varepsilon$ is close to 0 , that is, $q_{\varepsilon}$ is close to $q$. We will show that for any fixed $0 \leq \alpha<p N, \beta>0$, the minimizer of $R_{\alpha, \beta, \varepsilon}$ is non-radial provided that $\varepsilon$ is sufficiently small. 
LEMMA 4.1. If $N \geq 3$, there exists $c_{0}>0$, such that for every $q_{\varepsilon}$ and for every $0 \leq \alpha<p N, \beta>0$,

$$
c_{0} \beta^{\frac{p+1}{p\left(q_{\varepsilon}+1\right)}} \leq S_{\alpha, \beta, \varepsilon}^{r a d}
$$

Proof. From Lemma 2.1, we obtain

$$
\int_{\Omega}|x|^{\beta}|u(x)|^{q_{\varepsilon}+1} d x \leq C\left(\int_{\Omega}|x|^{-\frac{\alpha}{p}}|\Delta u|^{\frac{p+1}{p}} d x\right)^{\frac{p\left(q_{\varepsilon}+1\right)}{p+1}}
$$

where

$$
C=\left(\frac{1}{w_{N-1}}\right)^{\frac{p\left(q_{\varepsilon}+1\right)}{p+1}} \frac{1}{N+\beta-\left(N-2-\frac{N+\alpha}{p+1}\right)\left(q_{\varepsilon}+1\right)}\left(\frac{1}{N-2-\frac{N+\alpha}{p+1}}\right)^{q_{\varepsilon}+1}\left(\frac{1}{N+\alpha}\right)^{\frac{q_{\varepsilon}+1}{p+1}} .
$$

Since $u \in E_{\alpha}^{\mathrm{rad}}(\Omega)$ is arbitrary,

$$
c_{0}\left(N+\beta-\left(N-2-\frac{N+\alpha}{p+1}\right)\left(q_{\varepsilon}+1\right)\right)^{\frac{(p+1)}{p\left(q_{\varepsilon}+1\right)}} \leq S_{\alpha, \beta, \varepsilon}^{\mathrm{rad}},
$$

which ends the proof.

Let us denote by $S$ the classical Sobolev constant

$$
S=\inf _{u \in W^{2, \frac{p+1}{p}} \cap W_{0}^{1, \frac{p+1}{p}}(\Omega)} \frac{\int_{\Omega}|\Delta u|^{\frac{p+1}{p}} d x}{\left(\int_{\Omega}|u|^{q+1} d x\right)^{\frac{p+1}{p(q+1)}}} .
$$

It is standard that this Rayleigh quotient is invariant under translations and dilations.

LEMMA 4.2. If $N \geq 3$ and $0 \leq \alpha<p N, \beta>0$, then

$$
S=S_{\alpha, \beta, 0}<S_{\alpha, \beta, 0}^{r a d}
$$

Proof. Using Corollary 2.1, it is easy to verify that $S_{\alpha, \beta, 0}^{\mathrm{rad}}$ is achieved, so that $S<S_{\alpha, \beta, 0}^{\mathrm{rad}}$. Now, we claim that $S=S_{\alpha, \beta, 0}$. From the definition of $S_{\alpha, \beta, 0}$, we know that $S \leq S_{\alpha, \beta, 0}$. Thus, we will prove that $S_{\alpha, \beta, 0} \leq S$. Indeed, for $\delta>0$, we can choose $x_{\delta}=\left(1-\frac{1}{|\ln \delta|}, 0, \ldots, 0\right), U_{\delta}(x)=U\left(\frac{x-x_{\delta}}{\delta}\right)$ and $V_{\delta}(x)=V\left(\frac{x-x_{\delta}}{\delta}\right)$, where $(U, V)$ is the solution of (8). Let $\varphi_{\delta} \in C_{0}^{\infty}\left(\mathbb{R}^{N}\right)$ be a cut-off function satisfying

$$
\varphi_{\delta}(x)= \begin{cases}1, & x \in B\left(x_{\delta}, \frac{1}{2|\ln \delta|}\right), \\ 0, & x \in \mathbb{R}^{N} \backslash B\left(x_{\delta}, \frac{1}{|\ln \delta|}\right),\end{cases}
$$

$0 \leq \varphi_{\delta}(x) \leq 1,\left|\nabla \varphi_{\delta}(x)\right| \leq C|\ln \delta|,\left|\Delta \varphi_{\delta}\right| \leq C|\ln \delta|^{2} \quad$ in $\quad \mathbb{R}^{N}$, where $\quad C>0, \quad$ is independent of $\delta$. Set $w_{\delta}=\varphi_{\delta} U_{\delta}$, similar to [9], we have

$$
\lim _{\delta \rightarrow 0} \lim _{\varepsilon \rightarrow 0} \frac{\int_{\Omega}|x|^{-\frac{\alpha}{p}}\left|\Delta w_{\delta}\right|^{\frac{p+1}{p}} d x}{\left(\int_{\Omega}|x|^{\beta}\left|w_{\delta}\right|^{q_{\varepsilon}+1} d x\right)^{\frac{1}{q_{\varepsilon}+\frac{p+1}{p}}}} \leq S,
$$


and

$$
\begin{aligned}
& \lim _{\varepsilon \rightarrow 0} \frac{\int_{\Omega}|x|^{-\frac{\alpha}{p}}\left|\Delta w_{\delta}\right|^{\frac{p+1}{p}} d x}{\left(\int_{\Omega}|x|^{\beta}\left|w_{\delta}\right|^{q_{\varepsilon}+1} d x\right)^{\frac{1}{q_{\varepsilon}+1} \frac{p+1}{p}}} \\
& \geq \lim _{\varepsilon \rightarrow 0} \frac{\int_{\Omega}|x|^{-\frac{\alpha}{p}}\left|\Delta u_{\alpha, \beta, \varepsilon}\right|^{\frac{p+1}{p}} d x}{\left(\int_{\Omega}|x|^{\beta}\left|u_{\alpha, \beta, \varepsilon}\right|^{q_{\varepsilon}+1} d x\right)^{\frac{1}{q_{\varepsilon}+1} \frac{p+1}{p}}} \\
& \quad \geq \lim _{\varepsilon \rightarrow 0} \frac{\int_{\Omega}\left|\Delta u_{\alpha, \beta, \varepsilon}\right|^{\frac{p+1}{p}} d x}{\left(\int_{\Omega}\left|u_{\alpha, \beta, \varepsilon}\right|^{q+1} d x\right)^{\frac{1}{q+1} \frac{p+1}{p}}}
\end{aligned}
$$

where $u_{\alpha, \beta, \varepsilon}$ is a minimizer of $S_{\alpha, \beta, \epsilon}$; thus $S_{\alpha, \beta, 0} \leq S$.

Lemma 4.3. Assume that $N \geq 3$. For any $n \in \mathbb{N}$ there exists $\delta_{n}>0$ such that $S_{\alpha, \beta, \varepsilon}<$ $S_{\alpha, \beta, \varepsilon}^{\mathrm{rad}}$ provided $\beta \geq \frac{1}{n}$ and $\varepsilon<\delta_{n}$.

Proof. By contradiction, assume that there exists $n \in \mathbb{N}$ and sequences $\beta_{k} \geq \frac{1}{n}$ and $\delta_{k} \rightarrow 0$ such that

$$
S_{\alpha, \beta_{k}, \delta_{k}}=S_{\alpha, \beta_{k}, \delta_{k}}^{\mathrm{rad}}
$$

From Lemma 3.2, there exists $c_{1}$ independent of $q_{\delta_{k}}$, such that

$$
S_{\alpha, \beta, \delta_{k}} \leq c_{1} \beta_{k}^{\frac{2(p+1)}{p}-N+\frac{N(p+1)}{p\left(q_{\delta}+1\right)}} .
$$

Lemma 4.1 implies that

$$
c_{0} \beta_{k}^{\frac{p+1}{p\left(q \delta_{\delta_{k}}+1\right)}} \leq S_{\alpha, \beta, \delta_{k}}^{\mathrm{rad}}
$$

Since

$$
\frac{p+1}{p\left(q_{\delta_{k}}+1\right)}-\frac{2(p+1)}{p}+N-\frac{N(p+1)}{p\left(q_{\delta_{k}}+1\right)}=\frac{(p N-2 p-2)\left(q_{\delta_{k}}+1\right)-(p+1)(N-1)}{p\left(q_{\delta_{k}}+1\right)},
$$

we have

$$
\beta_{k}^{\frac{(p N-2 p-2)\left(q_{\delta_{k}}+1\right)-(p+1)(N-1)}{p\left(q_{\delta_{k}}+1\right)}} \leq \frac{c_{1}}{c_{0}} .
$$

From $q+1=\frac{N(p+1)}{N p-2 p-2}>\frac{(N-1)(p+1)}{N p-2 p-2}$ and $q_{\delta_{k}} \rightarrow q$ as $k \rightarrow+\infty$, it is implies that $(p N-2 p-2)\left(q_{\delta_{k}}+1\right)-(p+1)(N-1)>0$ as $k \rightarrow+\infty$. Thus, $\beta_{k}$ is bounded. We can assume that $\beta_{k} \rightarrow \beta \geq \frac{1}{n}$ as $k \rightarrow+\infty$.

Claim that

$$
S_{\alpha, \beta, 0}^{\mathrm{rad}}=\lim _{k \rightarrow+\infty} S_{\alpha, \beta_{k}, \delta_{k}}^{\mathrm{rad}}
$$

Indeed, by upper semi-continuity, it follows that

$$
S_{\alpha, \beta, 0}^{\mathrm{rad}} \geq \limsup _{k \rightarrow+\infty} S_{\alpha, \beta_{k}, \delta_{k}}^{\mathrm{rad}}
$$


On the other hand, from

$$
\int_{\Omega}|x|^{\beta_{k}}\left|u_{k}\right|^{q_{\delta_{k}}+1} d x \leq\left(\int_{\Omega}|x|^{\beta_{k}}\left|u_{k}\right|^{q+1} d x\right)^{\frac{q_{\delta_{k}}+1}{q+1}}\left(\int_{\Omega}|x|^{\beta_{k}} d x\right)^{\frac{q-q_{\delta_{k}}}{q+1}}
$$

we have

$$
S_{\alpha, \beta, 0}^{\mathrm{rad}} \leq \liminf _{k \rightarrow+\infty} S_{\alpha, \beta_{k}, \delta_{k}}^{\mathrm{rad}}
$$

Similarly, by upper continuity,

$$
S_{\alpha, \beta, 0} \geq \limsup _{k \rightarrow+\infty} S_{\alpha, \beta_{k}, \delta_{k}} .
$$

We obtain from (13)-(15), $S_{\alpha, \beta, 0} \geq S_{\alpha, \beta, 0}^{\mathrm{rad}}$, which contradicts Lemma 4.2.

\section{REFERENCES}

1. J. Byeon and Z-Q. Wang, On the Hénon equation: Asymptotic profile of ground state II, J. Differ. Equ. 216 (2005), 78-108.

2. J. Byeon and Z-Q. Wang, On the Hénon equation: Asymptotic profile of ground state I, Ann. Inst. H. Poincaré Anal. Non Linéaire 23 (2006), 803-828.

3. D. Cao and S. Peng, The asymptotic behavior of the ground state solutions for Hénon equation, J. Math. Anal. Appl. 278 (2003), 1-17.

4. X. Chen, C. Li and B. Ou, Classification of solution for a system of integral equations, Commun. Partial Differ. Equ. 30 (2005), 59-65.

5. D. G. De Figueiredo and P. L. Felmer, On superquadratic elliptic systems, Trans. Am. Math. Soc. 343 (1994), 99-106.

6. D. G. de Figueiredo, I. Peral and J. D. Rossi, The critical hyperbola for a hamiltonian elliptic system with weights, Ann. Mat. Pura Appl. 187 (2008), 531-545.

7. B. Gidas, W. M. Ni and L. Nirenberg, Symmetry of positive solutions of nonlinear elliptic equations in $\mathbb{R}^{N}$, in Mathematical analysis and applications: Part A, in Adv. in Math. Suppl. Stud., Vol. 7 (Academic Press, New York, 1981) pp. 369-402.

8. I. A. Guerra, Solutions of an elliptic system with a nearly critical exponent, Ann. Inst. H. Poincaré Anal. Non Linéaire 25 (2008), 181-200.

9. H. He and J. Yang, Asymptotic behavior of Solutions for Hénon systems with nearly critical exponent, J. Math. Anal. Appl. 347 (2008), 459-471.

10. M. Hénon, Numerical experiments on the stability of spherical stellar systems, Astronom. Astrophys. Lib. 24 (1973), 229-238.

11. J. Hulshof and R. C. A. M. van der Vorst, Differential systems with strongly indefinite variational struture, J. Funct. Anal. 114 (1993), 32-58.

12. J. Hulshof and R. C. A. M. van der Vorst, Asymptotic behavior of ground states, Proc. Am. Math. Soc. 124 (1996), 2423-2431.

13. F. Liu and J. Yang, Nontrivial solutions of Hardy-Hénon type elliptic systems, Acta Math. Sci. 27 (2007), 673-688.

14. W. M. Ni, A nonlinear Dirichlet problem on the unit ball and its applications, Indiana Univ. Math. J. 31 (1982), 801-807.

15. D. Smets, J. B. Su and M. Willem, Non-radial ground states for the Hénon equation, Comm. Contemp. Math. 4 (2002), 467-480.

16. D. Smets and M. Willem, Partial symmetry and asymptotic behavior for some elliptic variational problem, Calc. Var. Partial Differ. Equ. 18 (2005), 57-75. 